University of Nebraska - Lincoln

DigitalCommons@University of Nebraska - Lincoln

$8-2000$

\title{
Facile sol-gel synthesis of porous silicas using poly(propylene)imine dendrimers as templates
}

\author{
Gustavo F. Larsen \\ University of Nebraska - Lincoln, glarsen1@unl.edu \\ Edgar Lotero \\ University of Nebraska - Lincoln \\ Manuel Marquez \\ Los Alamos National Laboratory, NM
}

Follow this and additional works at: https://digitalcommons.unl.edu/cbmelarsen

Part of the Chemical Engineering Commons

Larsen, Gustavo F.; Lotero, Edgar; and Marquez, Manuel, "Facile sol-gel synthesis of porous silicas using poly(propylene)imine dendrimers as templates" (2000). Gustavo Larsen Publications. 1.

https://digitalcommons.unl.edu/cbmelarsen/1

This Article is brought to you for free and open access by the Chemical and Biomolecular Research Papers -- Faculty Authors Series at DigitalCommons@University of Nebraska - Lincoln. It has been accepted for inclusion in Gustavo Larsen Publications by an authorized administrator of DigitalCommons@University of Nebraska - Lincoln. 


\title{
Facile sol-gel synthesis of porous silicas using poly(propylene)imine dendrimers as templates
}

\author{
Gustavo Larsen ${ }^{\text {a) }}$ \\ Department of Chemical Engineering, University of Nebraska-Lincoln, \\ Lincoln, Nebraska 68588-0126 \\ Edgar Lotero \\ Department of Chemistry, University of Nebraska_Lincoln, Lincoln, Nebraska 68588-0304 \\ Manuel Marquez \\ Los Alamos National Laboratory, Chemical Science and Technology Division, \\ Los Alamos, New Mexico 87545, and Nanotechnology Laboratory (NanoteK), \\ Research and Development, Kraft Foods Inc., 801 Waukegan Road, Glenview, Illinois 60025
}

(Received 2 February 2000; accepted 5 June 2000)

Commercially available poly(propylene)imine (DAB-Am-32 and DAB-Am-64) dendrimers were used as single-molecule templates to tailor the porosity of silicas via a nonacidic sol-gel method. X-ray diffraction on both the as-prepared (oven-dried at $373 \mathrm{~K})$ and the calcined $(833 \mathrm{~K})$ materials revealed that modest contraction took place on template removal and that the cavities created did not achieve three-dimensional ordering under the current synthesis conditions. Transmission electron microscopy of "Pt-stained" samples supported this picture. A modified Horvath-Kawazoe analysis of the argon adsorption isotherms indicated that DAB-Am-64 is a much more effective template than DAB-Am-32. Pyrolysis and oxidation protocols for template removal are also presented.

\section{INTRODUCTION}

The use of micelles as templates to impart mesoporosity in silicas and aluminosilicates ${ }^{1,2}$ and other oxides ${ }^{3-8}$ is well documented. Based on the vast majority of available literature on the subject, it appears that alkylamine micelles are arguably the most suitable candidates for this task. From a fundamental viewpoint, it seems interesting to attempt similar materials syntheses using single polyamine-type macromolecules as porogenic agents. Molecular imprinting is a particularly difficult to task with inorganic substrates. There is some evidence for the imprinting of silica, ${ }^{9} \mathrm{Al}^{3+}$-doped silicas, ${ }^{10,11}$ and titania. ${ }^{12}$ Effective compounds to produce molecular imprints generally have stiff functional groups (aromatic, carbonyl); that is, multiple bonds that hinder internal rotations making the structure rather rigid and thus suitable for imprinting. Templates, on the other hand, are not required to produce molecular recognition sites. Rather, it is the generation of cavities and/or channels of controlled size that is considered sufficient to achieve "template" status.

This paper presents and discusses data on the use of poly(propylene)imine (DAB-Am-n) dendrimers as single-molecule porogens for sol-gel-derived silicas.

\footnotetext{
a) Address all correspondence to this author.
}

Based on an extensive literature search, we found no precedent for the use of DAB-Am-n dendrimers to produce silicas with controlled pore sizes. Poly(amidoamine)dendrimers (PAMAMs), on the other hand, have been used for self-assembly purposes in oxide, hybrid (organic/inorganic), and metallic materials. ${ }^{13-18}$ In fact, we have recently reported on the use of PAMAM 4.0 as a template to produce porous silicas. ${ }^{18}$ However, PAMAM 4.0 collapses upon heating, which results in pores that are significantly smaller than the PAMAM template diameter. ${ }^{18}$ Additives of small molecular sizes such as D-glucose have also been used effectively to impart mesoporosity in sol-gel materials. ${ }^{19}$ Very recently, Kriesel and Tilley ${ }^{20}$ produced carbosilane dendrimer sol-gels. The high silicon content of the dendrimers used by Kriesel and Tilley should prevent the generation of pores by high-temperature calcination, because $\mathrm{Si}$ atoms cannot be removed by oxidative thermal treatments. On the other hand, when a dendritic molecule that consists of only $\mathrm{C}, \mathrm{N}$, and $\mathrm{H}$ atoms is used as template, only gaseous products should be expected during the combustion process. Thus, our strategy is to use a silicon alkoxide, such as tetraethylorthosilicate (TEOS), as the Si source to generate the pore walls, and a combustible dendrimer as the template material.

Figure 1 shows a planar depiction of the so-called DAB-Am-32 poly(propylene)imine dendrimer. It has a 1-4 diaminobutane core (referred to as the "generation 0" 


\section{DAB-Am-32}

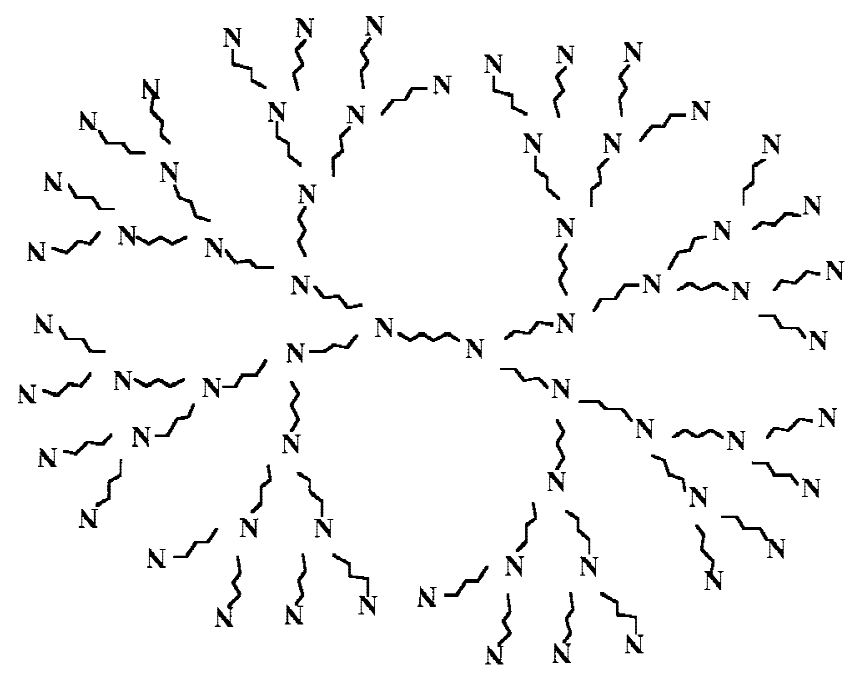

FIG. 1. A fourth-generation amine dendrimer structure (DAB-Am32). Outer $\mathrm{N}$-shell is saturated with hydrogen as terminal amino groups (not shown).

center), which branches out through its terminal nitrogen atoms via addiction of $-\left(\mathrm{CH}_{2}\right)_{3} \mathrm{~N}=$ difunctional building blocks. DAB-Am-32 is a "generation 4" poly(propylene)imine dendrimer; i.e., there are four generations of branches, and the outermost shell is composed of 32 primary amino groups. In a recent communication, we reported on the use of the largest commercially available member of this dendrimer family, namely DAB-Am-64, for the preparation of mesoporous silica with TEOS as the source of $\mathrm{Si}$, and $\mathrm{HCl}(0.12 \mathrm{~N})$ as the water/ hydrolysis-catalyst source. ${ }^{21}$ The DAB-Am-64 molecule having a total of $126 \mathrm{~N}$ atoms (64 primary amino groups in its outermost shell), the low acidity levels adopted in our original preparation protocol ${ }^{21}$ (approxiately one proton per dendrimer molecule) suggest that the hydrolysis of the $\mathrm{Si}$ alcoxide should proceed via basic catalysis, as a result of the intrinsic basicity of the soluble polyamine macromolecules. Thus, a synthetic route in which no acid is added was adopted in this study to confirm that similar materials can be synthesized without the aid of $\mathrm{HCl}$ or any other acid source. This contribution, in addition to demonstrating that DAB-Am-n-based mesoporous materials can be prepared in the absence of an acid catalyst, also introduces detailed characterization of the materials by mass spectrometry and electron microscopy. Both DAB-Am-32 and DAB-Am-64 are used as templates in this study, and the resulting pore diameters are discussed in light of x-ray diffraction (XRD), argon physisorption at low temperatures, and transmission electron microscopy (TEM) of "platinum-stained" samples. In this contribution, the pyrolysis and oxidation behavior of these new materials is also introduced for the first time.
One additional advantage of polyamine dendrimers that we will exploit in the future is that they are macrochelating agents for a number of metals with strong affinity for amine ligands, such as $\mathrm{Pt}, \mathrm{Cu}, \mathrm{Pd}$, $\mathrm{Co}$, etc. Thus, we view them as excellent vehicles for the synthesis of glasses with metal, or metal oxide nanoparticles with well-defined diameters.

\section{EXPERIMENTAL}

XRD measurements were carried out on a computerinterfaced Rigaku (The Woodlands, TX) instrument with a $\mathrm{Cu} \mathrm{K} \mathrm{K}_{\alpha}$ source. Electron microscopy studies were conducted in the bright-field mode with a JEOL JEM2010 (Sheboygan, WI) microscope at $200-\mathrm{keV}$ beam energy.

The Ar adsorption isotherms were obtained at $77 \mathrm{~K}$ on a computer-interfaced custom-built adsorption line at Porous Materials, Inc., Ithaca, NY. We did not perform desorption hysteresis studies because, as shown later, our materials have average pore sizes that are at the lower limit of those required for such analysis. Thus, a more accurate model that takes into account localized adsorption phenomena was adopted. Data analysis was done using the equations proposed by Chen and $\mathrm{Yang}^{22}$ in the form of a Fortran 77 code to model the adsorption of gases in spherical pores by a modified HorvathKawazoe $^{23}$ approach. A coverage-dependent term, as proposed by Chen and Yang, ${ }^{22}$ was incorporated into the modeling of spherical cavities. Polarizability and magnetic susceptibility data for both $\mathrm{Ar}$ and the oxide ion were taken from the literature. ${ }^{22}$ In our data analysis procedure, the only parameter that was slightly changed was the Ar polarizability (from $1.63 \times 10^{-24}$ to $1.0 \times$ $10^{-24} \mathrm{~cm}^{3}$ ) to reproduce accurate pore sizes of zeolite reference samples such as $5 \mathrm{~A}$ and $\mathrm{Y}$.

TEOS (1.02 g) was contacted with $1.60 \mathrm{~g}$ of a 1-propanol/DAB-Am-64 (or 1-propanol/DAB-Am-32) mixture consisting of $0.25 \mathrm{~g}$ of dendrimer per gram of solution. Anhydrous methanol $(0.57 \mathrm{~g})$ was also incorporated into the TEOS/dendrimer/1-propanol mixture prior to adding $0.25 \mathrm{~g}$ of deionized water. Once placed in a closed container at $343 \mathrm{~K}$, the aging time for this mixture was set at $12 \mathrm{~h}$. The resulting precipitate was oven dried at $373 \mathrm{~K}$ for $20 \mathrm{~h}$. Approximately $0.3 \mathrm{~g}$ of solid were heated in a $1 / 2$-in-inner diameter (ID) quartz U-tube under flowing nitrogen for $1 \mathrm{~h}$ at $803 \mathrm{~K}$. After allowing the sample to reach room temperature again in a $\mathrm{He}$ atmosphere, the solid was heated under flowing air from room temperature to $833 \mathrm{~K}$. This temperature was maintained for another $2 \mathrm{~h}$. The resulting white powders made from DAB-Am-64 and DAB-Am-32 were labeled as NU-1 and NU-2, respectively. Our selection of pretreatment temperatures was based on extensive preliminary work, by means of mass spectrometric detection of decomposition gases (as discussed in Sec. III), to address the 
issue of efficient removal of all carbonaceous matter from the materials surface without the unnecessary use of unreasonably high temperatures. For TEM analysis, about $30 \mathrm{mg}$ of the calcined materials were impregnated to incipient wetness with a saturated $\mathrm{H}_{2} \mathrm{PtCl}_{6}$ aqueous solution, and subsequently reduced under flowing hydrogen in a $1 / 4$-in-ID quartz U-tube at $723 \mathrm{~K}$ for $2 \mathrm{~h}$. The resulting (Pt-stained) powdered sample was suspended in isopropanol, and a drop of this suspension was allowed to evaporate onto a standard TEM holey carbon grid.

Temperature-programmed pyrolysis and oxidation (TPP and TPO) studies were conducted using a computer-interfaced MKS Residual Gas Analyzer. A total of $12 \mathrm{~m} / \mathrm{e}$ signals were followed The oven-dried powder $(50 \mathrm{mg})$ was placed in a $1 / 4$-in-ID quartz U-tube, and sandwiched between two quartz wool plugs. The quartz U-tube also contains a preheating bed comprised of low surface area, inert $\alpha-\mathrm{Al}_{2} \mathrm{O}_{3}\left(1-2 \mathrm{~m}^{2} / \mathrm{g}\right)$ granules placed upstream to the sample. Linear temperature ramps were applied. A K-type thermocouple was inserted through a septum secured by a Cajon-type fitting at the U-tube outlet. The tip of the thermocouple remained in contact with the outermost quartz wool plug, which is about $2 \mathrm{~mm}$ above the sample. Flowing ultrahigh-purity helium was used for the TPP cycle. The temperature was ramped from 298 to $803 \mathrm{~K}$ in $3 \mathrm{~h}$, followed by a plateau of $1 \mathrm{~h}$ at the same temperature. Instrument-grade air was used for the TPO cycle. This was carried out after the pyrolyzed sample was cooled to room temperature under He. On switching to air and flushing the sample flow cell for a few minutes, the temperature was ramped again from ambient to $833 \mathrm{~K}$ in $3 \mathrm{~h}$, and subsequently kept at the same value for another $2 \mathrm{~h}$. The TPP and TPO protocols mimic those used for the materials gas treatments, and their main goal is to allow us to gain some insight into the decomposition patterns of the template-loaded precursors.

\section{RESULTS AND DISCUSSION}

Figures 2 and 3 show the mass spectrometric analysis of the TPP and TPO gaseous effluent for these materials. The NU-2 and NU-1 materials produce remarkably similar TPP and TPO patterns, and it is therefore unnecessary to show results for the two TPP and the two TPO runs. Figure 2 shows all the detectable TPP signals.

After drying at $373 \mathrm{~K}$, a sol-gel material is normally expected to retain a fraction of unreacted alkoxy groups. ${ }^{24}$ This typically leads to reactive desorption of alkoxy moieties, as evidenced by $\mathrm{m} / \mathrm{e} 41$ (allyl cation fragment, olefins), and 28 (carbon monoxide). Water also accompanies the formation of olefins, because the former is a co-product of the alkoxy elimination process. The issue of TPP patterns of sol-gel-derived glasses and the $\mathrm{m} / \mathrm{e}$ assignments is discussed in more detail elsewhere. ${ }^{24}$

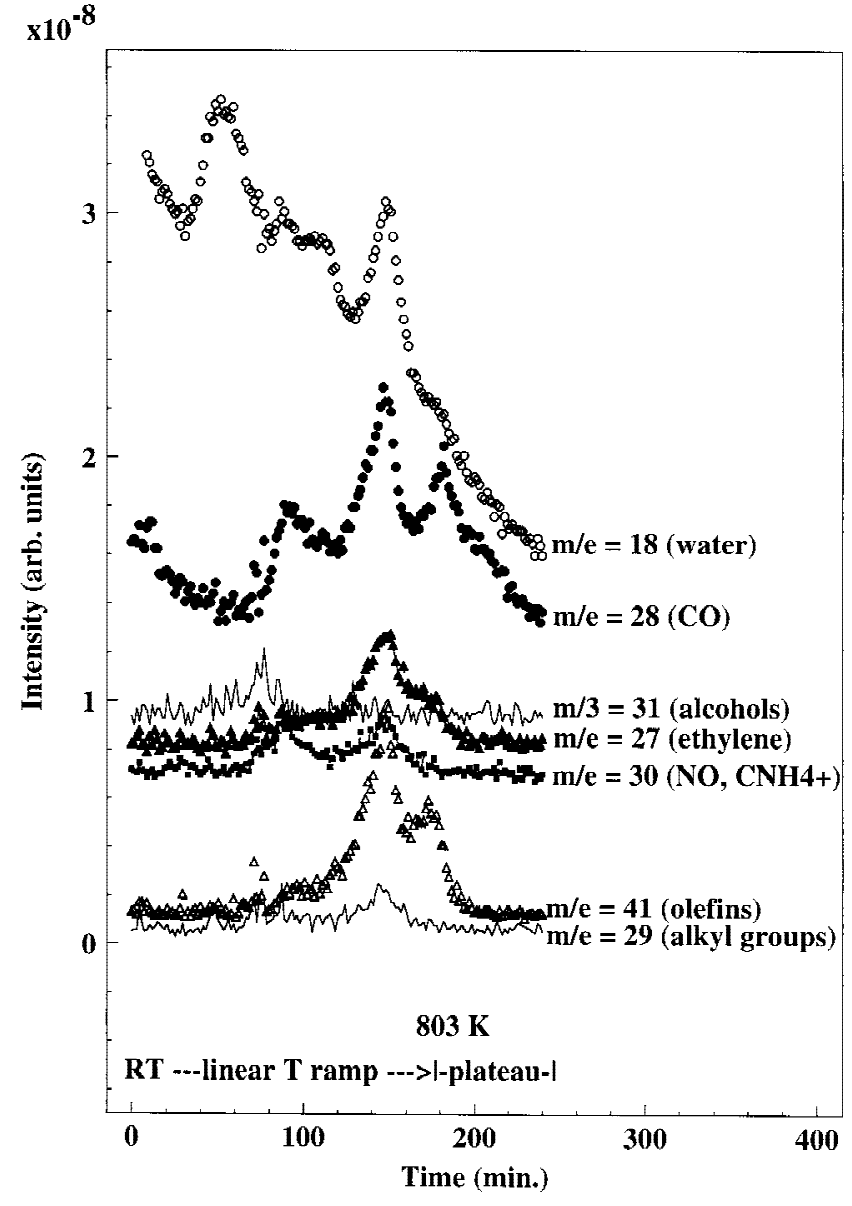

FIG. 2. Temperature-programmed pyrolysis of NU-2.

M/e 41 primarily tracks olefins larger than ethylene (this is shown by the occurrence of $\mathrm{m} / \mathrm{e} 27$ ). The sol-gel surface undergoes transetherification, despite the fact that the $\mathrm{Si}$ alkoxide precursor employed contains only ethoxy groups, because propanol and methanol are also present during gelation. Reversibly adsorbed water desorbs at around $470 \mathrm{~K}$, but it takes the full ramp-plateau cycle to produce a stable (pyrolyzed) material. Nonreactive alcohol desorption appears to be of relatively minor importance (m/e 31 is typically the strongest alcohol signal). M/e 30 is not representative of any conventional hydrocarbon cation formed at the mass spectrometer (MS) quadrupole. Rather, this species must correspond to either nitrous oxide, or to the $\mathrm{CH}_{2}=\mathrm{N}^{+} \mathrm{H}_{2}$ fragment from the dendritic structure. Nitrous oxide formation would involve a bimolecular decomposition process, because the only sources of oxygen atoms are the unreacted alkoxy moieties. Regardless of the identity of the desorbed nitrogen-containing species, our results indicate that nitrogen from the dendrimer template is lost prior to calcination. Desorption of saturated carbonaceous residues (m/e 29) seems rather negligible, which implies that olefin signals also arise from the decomposition of the dendrimer template. 
In summary, the chemical pathways shown below summarize our observations from Fig. 1 on the pyprolytic decomposition of our materials:

$\sim \mathrm{O}-\mathrm{CH}_{2}-\mathrm{CH}_{2}-\mathrm{R}$ (surface alkoxy groups, $\mathrm{R}:$ alkyl, $\left.\mathrm{H}\right) \rightarrow$

$$
\mathrm{CH}_{2}=\mathrm{CH}-\mathrm{R} \text { (olefins) , }
$$

$\sim \mathrm{O}-\mathrm{CH}_{2}-\mathrm{CH}_{2}-\mathrm{R}$ (surface alkoxy groups, $\mathrm{R}$ : alkyl, $\mathrm{H}$ ) $\rightarrow$

$\mathrm{CO}$ (from autoredox reactions) ,

DAB-Am-n dendrimer $\rightarrow \mathrm{CH}_{3} \mathrm{NH}_{2}$ and/or $\mathrm{NO}$

During the TPO cycle, only carbon dioxide and water were detected. Again, the full oxidation cycle is required to remove all carbon-containing matter. Pyrolysis and oxidation cycles involving lower temperatures are thus not recommended.

Figures 4 and 5 show the XRD patterns of NU-1 and NU-2 before and after calcination. The absence of higher-order reflections suggests that the formation of a three-dimensional arrangement of uniform cavities did not take place. On the other hand, it is interesting to compare the Bragg x-ray coherent lengths derived from

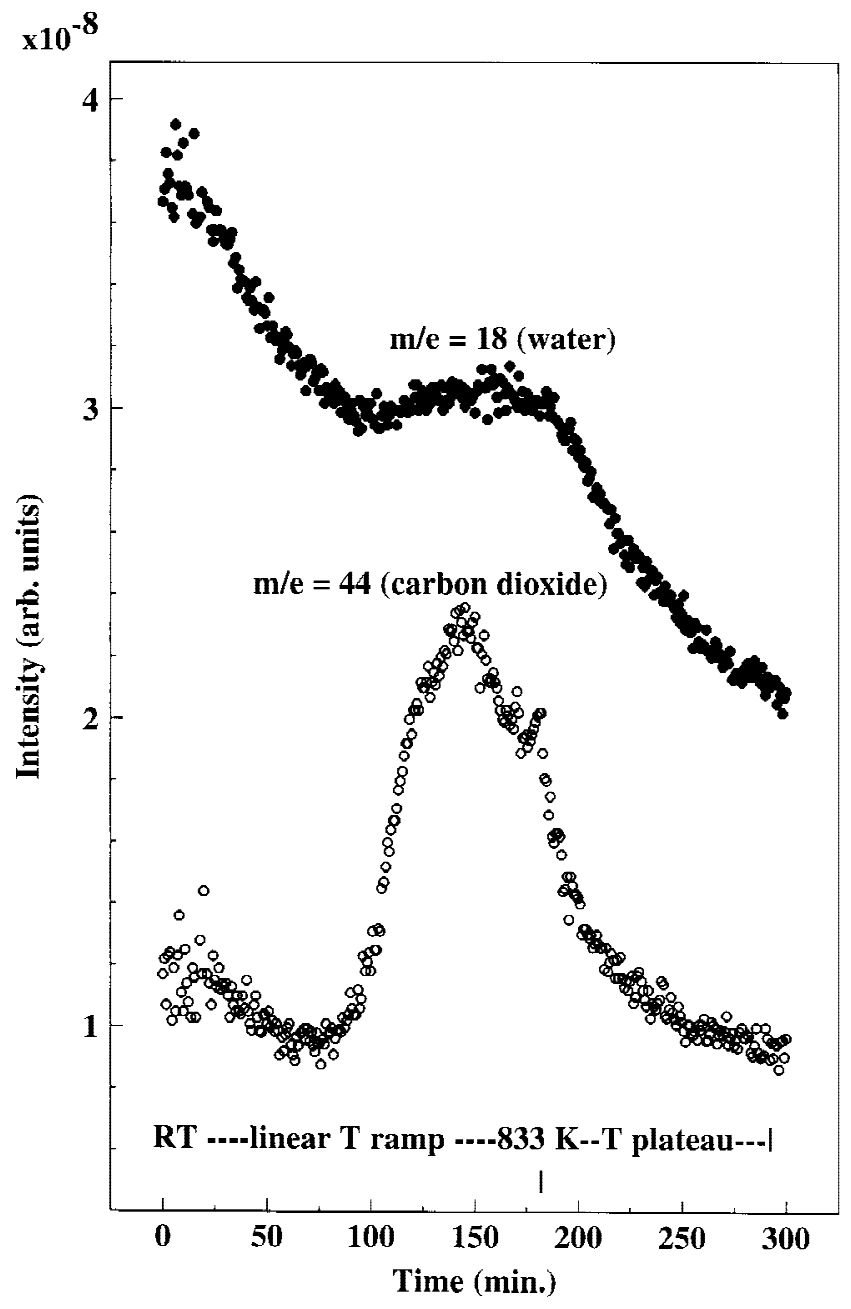

FIG. 3. Temperature-programmed oxidation of pyrolyzed NU-1. the centroids of the low-angle XRD reflections, and data on experimental and theoretical diameters of DAB molecules in diluted $\mathrm{D}_{2} \mathrm{O}$ solutions ${ }^{25}$ (see Table I). Unlike PAMAMs, DAB-Am-n dendrimers do not appear to aggregate in solution. 25 "Stretched-out," or "dense-shell,"

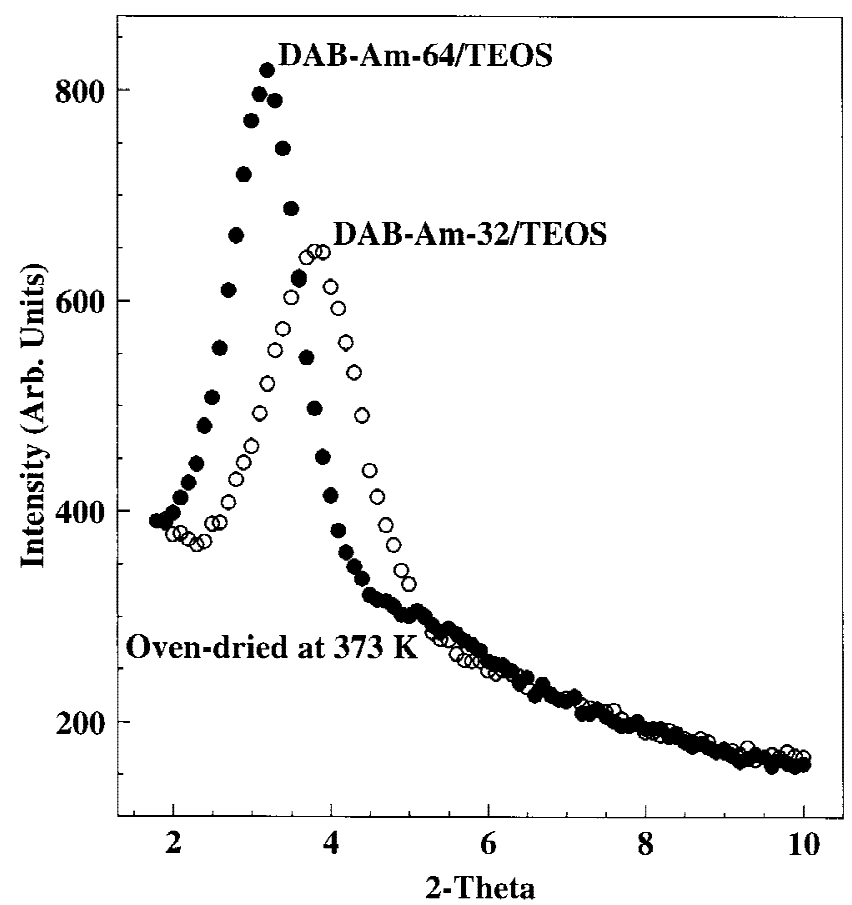

FIG. 4. XRD patterns of NU-1 and NU-2 prior to pyrolysis and calcination.

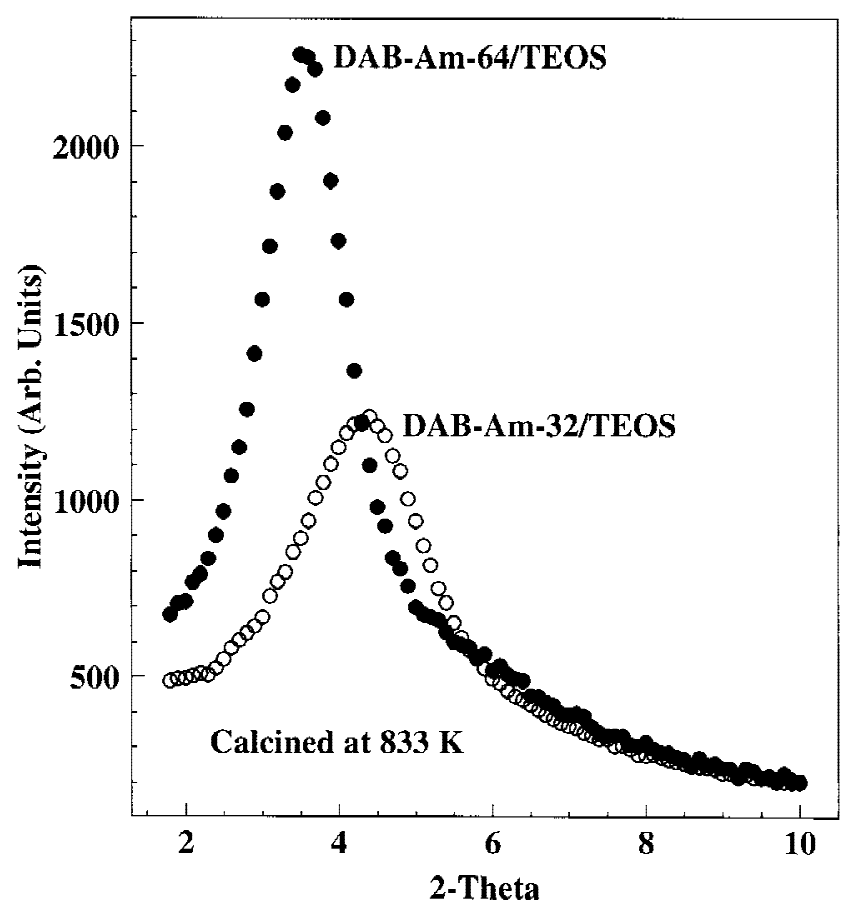

FIG. 5. XRD patterns of pyrolyzed NU-1 and NU-2 after calcination at $833 \mathrm{~K}$. 
TABLE I. Calculated XRD distances (L) and experimental and theoretical DAB-Am-32 and DAB-Am-64 diameter (D).

\begin{tabular}{lcccccc}
\hline \hline \multicolumn{1}{c}{ Material } & $\mathrm{L}_{373 \mathrm{~K}}$ & $\mathrm{~L}_{833 \mathrm{~K}}$ & $\mathrm{D}_{\mathrm{SANS}}{ }^{\mathrm{a}}$ & $\mathrm{D}_{\mu}{ }^{\mathrm{b}}$ & $\mathrm{D}_{\text {CVFFC }}{ }^{\mathrm{c}}$ & $\mathrm{D}_{\text {CVFFREP }}{ }^{\mathrm{d}}$ \\
\hline NU-1 & 27.7 & 24.8 & $\ldots$ & $\ldots$ & $\ldots$ & $\ldots$ \\
DAB-Am-64 & $\cdots$ & $\cdots$ & 27.8 & 39.6 & $\ldots$ & $\ldots$ \\
NU-2 & 23.4 & 20.3 & $\cdots$ & 31.2 & $\ldots$ & $\ldots$ \\
DAB-Am-32 & $\cdots$ & $\cdots$ & 23.2 & $\ldots .0$ & 20.0 \\
\hline \hline
\end{tabular}

All parameters reported in angstroms. Experimental values for pure dendrimers are in diluted $\mathrm{D}_{2} \mathrm{O}$ solutions.

${ }^{\text {a}}$ Small-angle neutron scattering, from Ref. 25.

${ }^{\mathrm{b}}$ Hydrodynamic (viscosity) measurements, from Ref. 25.

${ }^{c}$ Consistent valence force field (CVFF) in which both coulombic and van der Waals interactions between nonbonding atoms are considered (data from Ref. 25).

${ }^{\mathrm{d}} \mathrm{CVFF}$ in which only repulsive forces between nonbonding atoms are considered (data from Ref. 25 ).

conformations are obtained by molecular-dynamics simulations at the consistent valence force field "repulsive" (CVFFREP) level of theory. Figure 1 is indeed a dense-shell representation of DAB-Am-32; i.e., the dendrimer branches extend toward the "shell," or the outer surface of the molecule, reaching their maximum length because their $\mathrm{C}, \mathrm{N}$ backbones adopt an all-anti conformation. The net effect is that terminal groups crowd the shell. On the contrary, when extensive back folding of the branches takes place, the dendrimer molecule decreases its diameter and increases its density toward the center. The so-called "dense core" structure is thus formed.

The CVFFREP results shown in Table I from Ref. 25 only consider repulsive forces between nonbonding atoms. On the other hand, structures much closer to the so-called dense core limit arise from calculations using the consistent valence force field "complete" (CVFFC) approach. Indeed, rather broad radial density distribution functions for these dendritic species are predicted by theory. ${ }^{25}$ This is a key property of DAB-Am-n dendrimers; it is likely impossible that the sol-gel-trapped dendrimers adopt either a dense shell or a dense core conformation exclusively. Thus, the flexibility of dendrimers should ultimately broaden the pore-size distributions (PSDs) of the calcined materials. However, it appears that once trapped in a sol-gel matrix, conformations close to the dense core limit are preferred, judging from our XRD and adsorption (see below) results. Experimental data on the effective diameter of these two dendrimers in solution ${ }^{25}$ also cluster at or above the predicted XRD distances in the NU-1 and NU-2 materials that still have the dendritic molecules trapped in the alcogel. The importance of a comparison between theoretical and experimental data on DAB-Am-n diameters in solution, and XRD coherent lengths and pore diameters, lies in that the exercise represents a check for the ability of these molecules to utilize their molecular volume effectively to imprint well-defined cavities of in oxide materials. On pyrolysis and calcination, gel densification and loss of organic matter appears to cause further shrinkage, and XRD predicts that cavities with diameter around $3 \AA$ smaller than those of the trapped dendrimers are formed. Interestingly, we noticed no significant difference in light of XRD data between this NU-1 preparation, and that reported earlier using $\mathrm{HC} 1$ as catalyst for alkoxide hydrolysis. As mentioned above, the influence of adding roughly one proton per dendrimer molecule on template diameter is expected to be very small. A broad XRD signal around $2 \theta \sim 22^{\circ}$ (not shown) is indicative of the amorphous nature of these materials.

The Ar Brunauer-Emmett-Teller analysis (BET)specific surface areas of NU-1 and NU-2 were found to be 767.4 and $585.1 \mathrm{~m}^{2} / \mathrm{g}$, respectively. A blank material (i.e., a sample prepared in the absence of dendrimers) had a BET-specific surface area of $241.0 \mathrm{~m}^{2} / \mathrm{g}$, and a very broad pore-size distribution in the 8 to $16 \AA$ range. The $\mathrm{Ar}$ adsorption isotherms, and the modified HorvathKawazoe PSD curves for NU-1 and NU-2 are shown in Figs. 6 and 7, respectively. The scattering of data in the first derivative curves is due to the fact that a good number of isotherm data points were taken too close to each other, thereby exaggerating the error associated with differentiation. Nevertheless, two features in Fig. 7 are immediately apparent. First, for Ar adsorption under the adopted modified Horvath-Kawazoe formalism, the PSD of NU-1 peaks at about $27 \AA$. This is approximately $2 \AA$ above the value predicted by XRD, but it must be realized that the constraint imposed by the requirement of strict adherence of the adsorption data to a spherical pore model could well be responsible for this small discrepancy. Second, NU-2 has a broader PSD, which roughly centers at about $17 \AA$. There is apparently little doubt that the largest dendrimer effects the formation of mesosized cavities. The PSD curve of NU-1 lies well above those expected for microporous silicas, which are normally centered around $16 \AA .{ }^{26}$ On the other hand, the breath of the PSD of NU-2 suggests that DAB-Am-32, being more flexible than its larger counterpart, with a sterically less-impeded (crowded) outermost shell, is relatively less effective as template under the current synthesis conditions. The net effect of adding one more "generation" of branches to DAB-Am-32 (see Fig. 1) to form DAB-Am-64 would be the crowding or densification of the outer shell. The low-angle XRD pattern of 


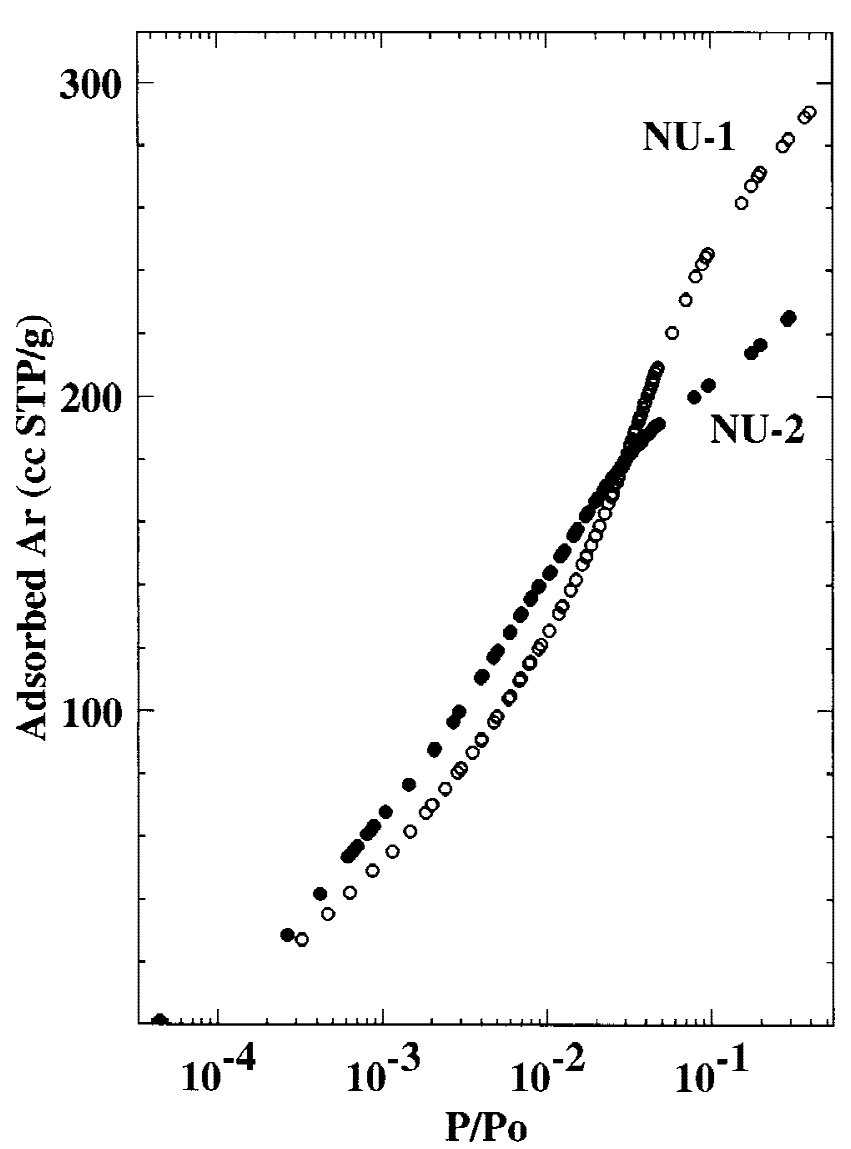

FIG. 6. Ar adsorption isotherms of calcined NU-1 and NU-2 at $77 \mathrm{~K}$.

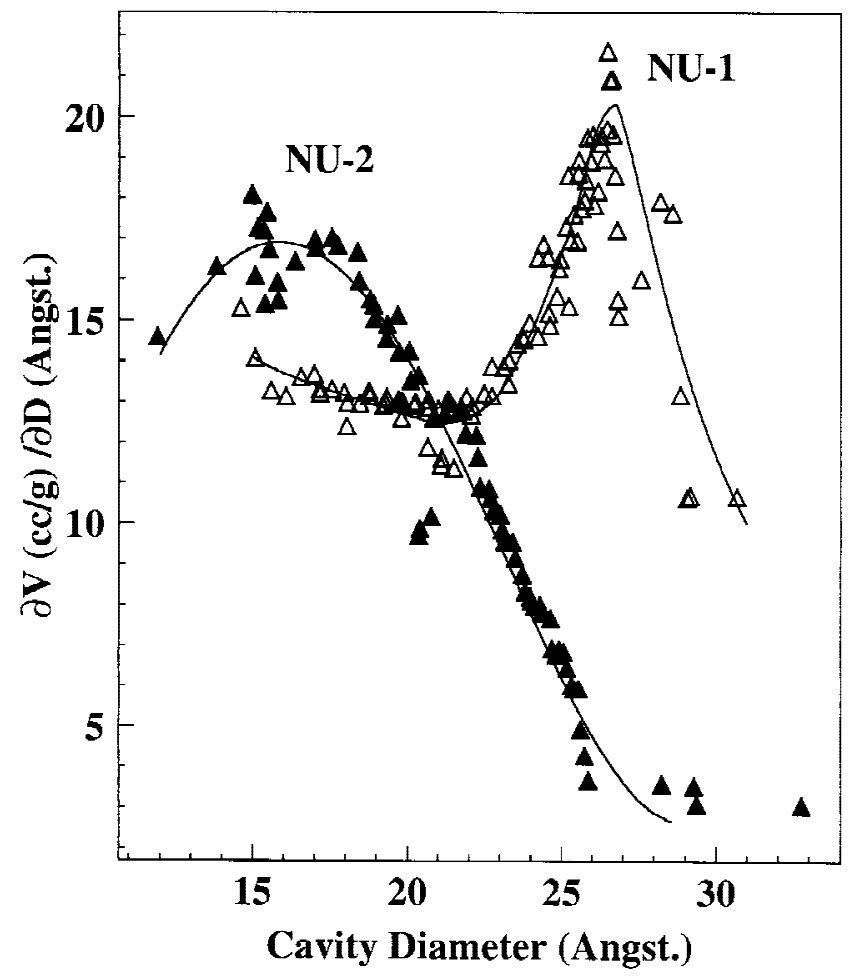

FIG. 7. Modified Horvath-Kawazoe PSDs of calcined NU-1 and NU-2.
NU-2 probes only a fraction of the pores, namely those of uniform size, which are the only ones capable of generating the occurrence of x-ray coherent lengths. In addition, note that the low-angle XRD peak in NU-2 is weaker and broader than that of NU-1, both before and after calcination. Figure 8 suggests an explanation for this observation: unlike NU-1, the PSD of NU-2 is not necessarily dominated by the template.

A representative TEM image of Pt-loaded NU-1 is shown in Fig. 8. The NU-1 particles were found to be globular, with diameters in the order of 200 to $500 \mathrm{~nm}$. As mentioned earlier, ${ }^{21}$ unless a high-Z element "stain" is used, it is very difficult to obtain information about the pore structure of these materials by TEM. The cavities generated by the template effect would have to align in a single file to form regions of high contrast. This was not found to be the case. In our previous work, ${ }^{21}$ we incorporated $\mathrm{Cu}$ ions during synthesis and TEM imaged the

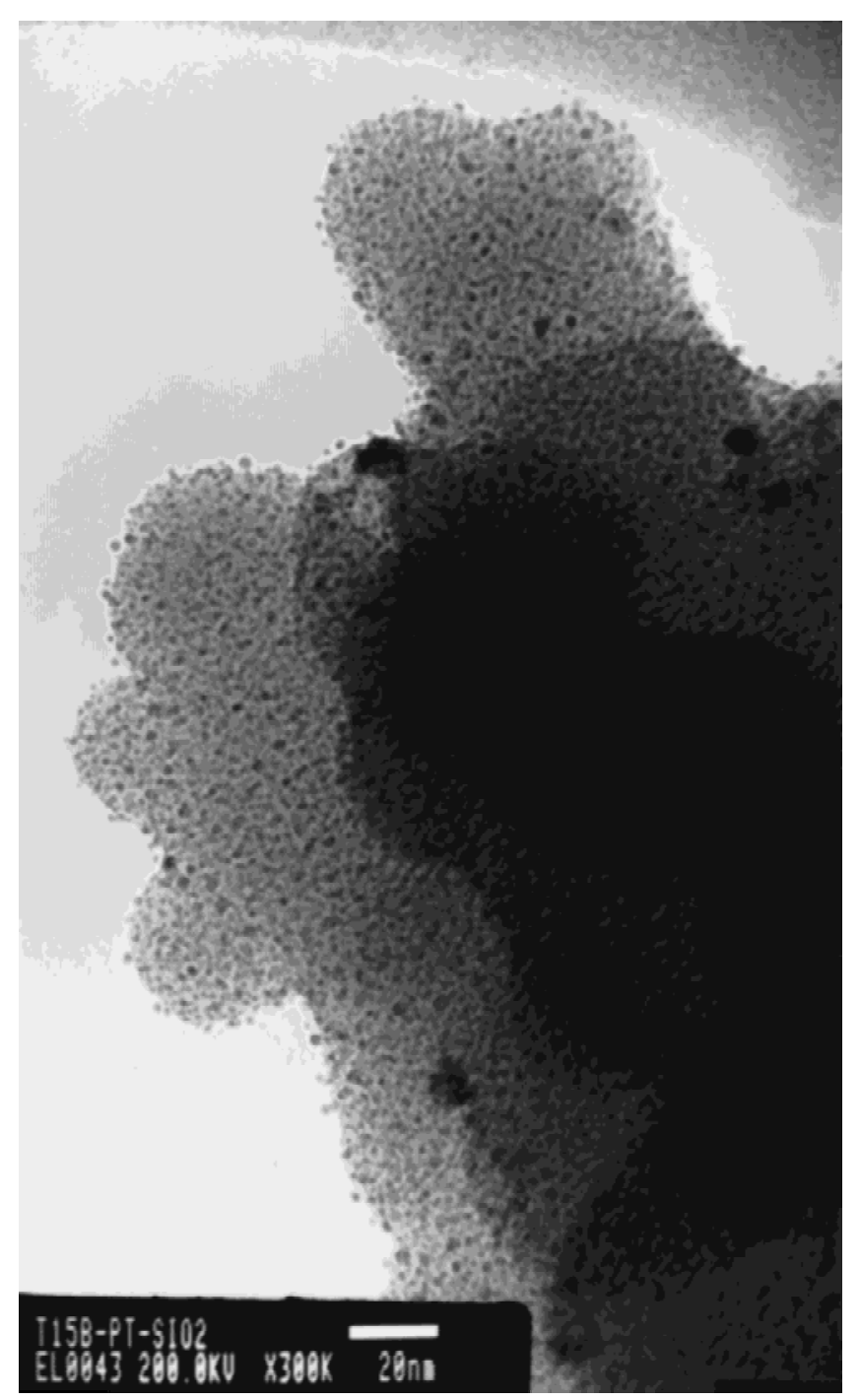

FIG. 8. TEM image of Pt-loaded (calcined) NU-1. 
oven-dried alcogel, which showed highly uniform $\mathrm{Cu}_{\mathrm{n}}{ }^{\mathrm{m}+} /$ dendrimer complexes trapped in the NU-1 matrix. In this work, we have used a different approach for a few reasons, the most important one being our desire to image the materials after calcination. In Fig. 8, trapped Pt clusters of about the same size as those of the cavity diameters derived from XRD and physisorption measurements are observed. In a few cases (not shown), we observed the formation of a small number of larger $\mathrm{Pt}$ particles that had migrated out of the pores and were the result of relatively unimpeded surface mobility and sintering. Nevertheless, the pore network in these materials should be relatively open because pyrolysis and oxidation products, as well as Pt species, appear to have diffused well through the NU-1 gel particles.

\section{CONCLUSIONS}

In conclusion, a nonacidic sol-gel route for the synthesis of dendrimer-mediated mesoporous silica is demonstrated. To produce a mesoporous material from DAB-Am-64 with a disordered network of rather uniform pores, effective pyrolytic and oxidative decomposition cycles for the resulting dendrimer/sol-gel composites are described. The calcined materials are ideal guests for well-dispersed transition metal clusters, which can be produced by conventional incipient wetness of metal precursors, followed by chemical reduction under hydrogen at high temperatures. The potential of dendrimer-loaded sol-gels (i.e., prior to template removal by calcination) as carriers for occluded metal ion aggregates (synthesis of glasses with optical and magnetic properties, heterogeneous catalysts, etc.), the use of hydrothermal (zeolitelike) materials synthesis routes, and the extent of pore connectivity, are all important issues that remain to be addressed.

\section{ACKNOWLEDGMENTS}

This work was supported by the National Science Foundation (CTS-9733756) and a Kraft Foods Inc. research grant.

\section{REFERENCES}

1. J.S. Beck, J.C. Vartuli, W.J. Roth, M.E. Leonowicz, C.T. Kresge, K.D. Schnitt, C.T-W. Chu, D.H. Olson, E.W. Sheppard, S.B. McCullen, J.B. Higgins, and J.L. Schlenker, J. Am. Chem. Soc. 114, 10834 (1992).

2. C.T. Kresge, M.E. Leonowicz, W.J. Roth, J.C. Vartuli, and J.S. Beck, Nature 359, 710 (1992).

3. E. Zhao, O. Hernandez, G. Pacheco, S. Hardcastle, and J.J. Fripiat, J. Mater. Chem. 8, 1635 (1998).

4. Y-Y. Huang, T.J. McCarthy, and W.M.H. Sachler, Appl. Catal. A 163, 245 (1997).

5. V.F. Stone and R.J. Davis, Chem. Mater. 10, 1468 (1998).

6. D.M. Antonelli and J.Y. Ying, Angew. Chem. 34, 2014 (1995).

7. G. Larsen, E. Lotero, M. Nabity, L.M. Petkovic, and D.S. Shobe, J. Catal. 164, 246 (1996).

8. W. Zhang, M. Froba, J. Wang, P.T. Tanev, J. Wong, and T.J. Pinnavaia, J. Am. Chem. Soc. 118, 9164 (1996).

9. A. Katz and M.E. Davis, Nature 403, 286 (2000).

10. K. Morihara, S. Kurihara, and J. Suzuki, Bull. Chem. Soc. Jpn. 61, 3991 (1988).

11. K. Morihara, M. Takiguchi, and T. Shimada, Bull. Chem. Soc. Jpn. 67, 1078 (1994).

12. S-W. Lee, I. Ichinose, and T. Kunitake, Langmuir 14, 2857 (1998).

13. Y. Chujo, H. Matsuki, S. Kure, T. Saegusa, and T. Yazawa, J. Chem. Soc. Chem. Commun. 635 (1994).

14. H. Fan, Y. Zhou, and G.P. Lopez, Adv. Mater. 9, 728 (1997).

15. V.V. Kazakova, V.D. Myakushev, T.V. Strelkova, N.G. Gvazava, and A.M. Muzafarov, Dokl. Akad. Nauk 349, 486 (1996).

16. J.L. Hendrick, C.J. Hawker, M. Trollsas, J. Remenar, R. Yoon, and R.D. Miller, in Flat-Panel Display Materials -1998, edited by G.N. Parsons, C-C.Tsai, T.S. Fahlen and C.H. Seager (Mater. Res. Soc. Symp. Proc. 519, Warrendale, PA, 1998), p. 65.

17. L. Balogh, A. de Leuze-Jallouli, P.R. Dvornic, M.J. Owen, S.V. Sperz, and R. Spindler, U.S. Patent No. 5938934 (17 August 1999).

18. G. Larsen, E. Lotero, and M. Marquez, J. Phys. Chem. B 104, 4840 (2000).

19. Y. Wei, J. Xu, H. Dong, J.H. Dong, K. Kiu, and S.A. JansenVarnum, Chem. Mater. 11, 2023 (1999).

20. J.W. Kriesel and T.D. Tilley, Chem. Mater. 11, 1190 (1999).

21. G. Larsen, E. Lotero, and M. Marquez, Chem. Mater. 12, 1513 (2000).

22. L.S. Cheng, and R.T. Yang, Chem. Eng. Sci. 49, 2599 (1994).

23. G. Horvath and K. Kawazoe, J. Chem. Eng. Jpn. 16, 470 (1983).

24. E. Lotero, D. Vu, C. Nguyen, J. Wagner, and G. Larsen, Chem. Mater. 10, 3756 (1998).

25. R. Scherrenberg, B. Coussens, P. van Vliet, G. Edouard, J. Brackman, E. de Brabander, and K. Mortensen, Macromolecules 31, 456 (1998).

26. C.J. Brinker and G.W. Scherer, J. Non-Cryst. Solids 70, 301 (1985). 